\title{
AN INTEGRAL FORMULA FOR COMPACT HYPERSURFACES IN A EUCLIDEAN SPACE AND ITS APPLICATIONS \\ by SHARIEF DESHMUKH
}

(Received 3 April 1991; revised 18 October, 1991)

1. Introduction. Let $M$ be a compact hypersurface in a Euclidena space $\mathbb{R}^{n+1}$. The support function $\rho$ of $M$ is the component of the position vector field of $M$ in $\mathbb{R}^{n+1}$ along the unit normal vector field to $M$, which is a smooth function defined on $M$. Let $S$ be the scalar curvature of $M$. The object of the present paper is to prove the following theorems.

THEOREM 1. Let $M$ be a compact hypersurface of $\mathbb{R}^{n+1}$ with non-negative Ricci curvature. Then

$$
\operatorname{Av}(S) \geq n(n-1) / \operatorname{diam}^{2}(M),
$$

where $\operatorname{Av}(S)$ is the average scalar curvature of $M$ given by the Einstein functional $\operatorname{Av}(S)=1 / \operatorname{vol}(M) \int_{M} S d v$, and $\operatorname{diam}(M)$ is the diameter of $M$.

THEOREM 2. Let $M$ be a compact hypersurface of $\mathbb{R}^{n+1}$ with non-negative Ricci curvature. If $M$ is centrally symmetric and $R$ is the radius of the escribed sphere, then $R^{2} \geq n(n-1) / \operatorname{Av}(S)$.

THEOREM 3. Let $M$ be a compact and connected hypersurface of positive Ricci curvature in $\mathbb{R}^{n+1}$. If the support function $\rho$ of $M$ satisfies $\rho^{2} \leq n(n-1) / S$, then $\rho$ is a constant and $M$ is a sphere of radius $\rho$.

THEOREM 4. Let $M$ be a compact and connected hypersurface of non-negative Ricci curvature in $\mathbb{R}^{n+1}$. If $M$ is contained in a closed ball of radius $R$ centered at origin in $\mathbb{R}^{n+1}$ and the scalar curvature $S$ of $M$ satisfies sup $S=n(n-1) R^{-2}$, then $M$ is the sphere of radius $R$.

All above theorems are consequences of an integral formula which we prove in Section 2. We observe that Theorem 4 generalizes Theorem 1 in [1] for hypersurfaces of non-negative Ricci curvature in a Euclidean space. We also get the following corollary to Theorem 1 which generalizes the result of Jacobwitz [2] for non-immersibility of a compact Riemannian manifold of non-negative Ricci curvature into a closed ball in a Euclidean space.

COROLlary. Let $M$ be a compact n-dimensional Riemannian manifold of nonnegative Ricci curvature whose average scalar curvature satisfies $\operatorname{Av}(S)<n(n-1) R^{-2}$. Then no isometric immersion of $M$ into $\mathbb{R}^{n+1}$ is contained in a closed ball of radius $R$ in $\mathbb{R}^{n+1}$.

We express our sincere thanks to Referee for many helpful suggestions.

2. Integral formula. Let $M$ be a compact hypersurface in $\mathbb{R}^{n+1}$ and $N$ be the globally defined unit normal vector field on $M$. We denote by $g, \nabla$ and $A$, the induced metric, the covariant derivative operator with respect to the induced Riemannian

This work is supported by the research grant No. (Math/1409/05) of the Research Center, College of Science, King Saud University, Riyadh.

Glasgow Math. J. 34 (1992) 309-311. 
connection and the shape operator on $M$. Then we have

$$
\bar{\nabla}_{X} Y=\nabla_{X} Y+g(A X, Y) N, \quad \bar{\nabla}_{X} N=-A X, \quad X, Y \in \mathscr{L}(M),
$$

where $\bar{\nabla}$ is the covariant derivative operator with respect to the Euclidean connection on $\mathbb{R}^{n+1}$ and $\mathscr{L}(M)$ is the Lie algebra of vector fields on $M$. Let $T$ be the position vector field on $\mathbb{R}^{n+1}$. Then the smooth function $\rho: M \rightarrow R$ defined by $\rho=\left\langle\left. T\right|_{M}, N\right\rangle$ is called the support function of the hypersurface $M$, where $\langle$,$\rangle is the Euclidean metric on \mathbb{R}^{n+1}$. We have $\left.T\right|_{M}=\xi+\rho N, \xi \in \mathscr{X}(M)$. Then since $\bar{\nabla}_{X} T=X$ holds for any $X \in \mathscr{X}(M)$, using (2.1), we have

$$
\nabla_{X} \xi=X+\rho A X, \quad d \rho(X)=-g(A X, \xi), \quad X \in \mathscr{L}(M) .
$$

From the equation of Gauss for hypersurface $M$ in $\mathbb{R}^{n+1}$, we get the following expressions for Ricci curvature and scalar curvature $S$ of $M$ (cf. [3])

$$
\begin{gathered}
\operatorname{Ric}(X, Y)=n \alpha g(A X, Y)-g(A X, A Y), \quad X, Y \in \mathscr{X}(M), \\
S=n^{2} \alpha^{2}-\operatorname{tr} A^{2},
\end{gathered}
$$

where $\alpha=1 / n \sum g\left(A e_{i}, e_{i}\right)$ is the mean curvature of $M$ and $\left\{e_{1}, \ldots, e_{n}\right\}$ is a local orthonormal frame on $M$.

LEMMA 2.1 .

$$
\int_{M}\left\{\operatorname{Ric}(\xi, \xi)+n(n-1)-\rho^{2} S\right\} d v=0 .
$$

Proof. We use equation (2.2) to compute the Laplacian of the support function $\rho$ and the divergence of the vector field $\xi$, and obtain

$$
\Delta \rho=-n d \alpha(\xi)-n \alpha-\rho \operatorname{tr} A^{2}, \quad \operatorname{div} \xi=n(1+\rho \alpha) .
$$

Integrating second equation over $M$ we get

$$
\int_{M}(1+\rho \alpha) d v=0
$$

Also we have

$$
-n \rho d \alpha(\xi)=n \alpha \operatorname{div}(\rho \xi)-\operatorname{div}(n \alpha \rho \xi)=n \alpha d \rho(\xi)+n \rho \alpha \operatorname{div} \xi-\operatorname{div}(n \alpha \rho \xi) .
$$

Using second equation in (2.2) and above equation in (2.5), we find

$$
\rho \Delta \rho=-n \alpha g(A \xi, \xi)+n^{2} \rho \alpha+n^{2} \rho^{2} \alpha^{2}-n \rho \alpha-p^{2} \operatorname{tr} A^{2}-\operatorname{div}(n \alpha \rho \xi) .
$$

We find $\operatorname{grad} \rho=-A \xi$ from equation (2.2) and use it together with equation (2.7) in $\frac{1}{2} \Delta \rho^{2}=\rho \Delta \rho+\|\operatorname{grad} \rho\|^{2}$ to obtain

$$
\frac{1}{2} \Delta \rho^{2}=-\operatorname{Ric}(\xi, \xi)-n(n-1)+n(n-1)(1+\rho \alpha)+\rho^{2} S-\operatorname{div}(n \alpha \rho \xi),
$$

where we have also used equation (2.3) and (2.4). Integrating above equation over $M$ and using integral formula (2.6), we get the desired result.

3. Proofs of theorems. Theorems 1 and 2 follow directly from Lemma 2.1, as their hypotheses give

$$
\operatorname{diam}^{2}(M) \int_{M} S d v \geq \int_{M} \rho^{2} S d v \geq n(n-1) \operatorname{vol}(M)
$$


For Theorem 3, we observe that Lemma 2.1 gives $\xi=0$, which when combined with equation (2.2) gives $\rho$ is a constant. That $\rho$ is a non-zero constant is guarnateed by integral formula (2.6). Thus in this case from first equation in (2.2), we have that $M$ is a totally umbilic and hence a sphere of radius $\rho$ (cf. [3], p. 30).

The hypothesis of Theorem 4 confirms that $R^{2} S \leq n(n-1)$. Now using a unit vector field $t=\xi /\|\xi\|$, defined on the open subset of $M$ where $\xi$ is non-zero, and $\left\|\left.T\right|_{M}\right\|^{2}=$ $\|\xi\|^{2}+\rho^{2}$ in Lemma 2.1 , we obtain

$$
\int_{M}\left\{\|\xi\|^{2}(\operatorname{Ric}(t, t)+S)+\left(n(n-1)-\left\|\left.T\right|_{M}\right\|^{2} S\right)\right\} d v=0 .
$$

Since $M$ lies in a closed ball of radius $R$ in $\mathbb{B}^{n+1}$, we may assume that $\left\|\left.T\right|_{M}\right\|^{2} \leq R^{2}$ and consequently we have

$$
n(n-1)-\left\|\left.T\right|_{M}\right\|^{2} S \geq n(n-1)-R^{2} S \geq 0,
$$

where we have used $S \geq 0$ which follows from the hypothesis. Thus the above integral together with the inequality above gives $\xi=0$ and $\left\|\left.T\right|_{M}\right\|^{2}=R^{2}=\rho^{2}$. This proves the theorem.

\section{REFERENCES}

1. L. Coghlan and Y. Itokawa, On the sectional curvature of compact hypersurfaces, Proc. Amer. Math. Soc. 109(1) (1990), 215-221.

2. H. Jacobowitz, Isometric embedding of a compact Riemannian manifold into Euclidean space, Proc. Amer. Math. Soc. 40(1) (1973), 245-246.

3. S. Kobayashi and K. Nomizu, Foundations of differential geometry vol. II (Interscience Publ., 1969).

Department of Mathematics

College of Science

King Saud University

P.O. Box 2455

RIYADH 11451

Saudi ARABia 